\title{
Celiac Disease: Far from Promised Land
}

\author{
Rocío Aguado $^{1}$, Orlando Estevez ${ }^{1}$, Silvia Fernández ${ }^{2}$, Consuelo Ortega ${ }^{2}$, Manuel Santamaría ${ }^{1,2, *}$ \\ ${ }^{1}$ Clinical Immunology Service, Reina Sofía University Hospital, Córdoba, Spain \\ ${ }^{2}$ Department of Immunology, School of Medicine, University of Córdoba. Córdoba, Spain \\ *Corresponding author: msantamaria@uco.es
}

The diagnostic resources currently available in the first world countries have greatly improved with serological test, mainly anti-endomysium and anti-tissuetransglutaminase 2 autoantibodies detection. Nevertheless, identification of non-typical patients in serological terms remains as one of the main task to be afforded. In this sense, new biomarkers are currently emerging and should receive close attention. Among others has recently been observed that the regenerating gene I alpha protein, involved in tissue regeneration, is elevated in the sera of celiac patients and in their targeted tissue during tissue inflammation and damage, decreasing after gluten-free diet. Other significant example of a potential new biomarker in celiac disease consist of the intestinal fatty acid binding protein (I-FABP), whose levels are elevated in the celiac disease. Serum levels of I-FABP seems to correlate with the severity of enterocyte damage. Also a bunch of autoantibodies are arising over the skyline of celiac disease diagnose. Thus, IgA isotype anti-actin antibodies (IgA-AAA) presence, are somehow related to intestinal villous atropy in celiac disorder. Indeed, severe intestinal damage is observed in patients where IgA-AAA autoantibodies. Recently, ESPAGHAN included in its guidelines the detection of anti-deamidated gliadin peptide (DPG) antibodies that has been shown to be very efficient in the identification of the disease in children younger than 2 years-old. Failure to thrive usually prompts initial studies for celiac disease. In the absence of celiac disease markers, the differential diagnose with other causes leading to thriving failure is not always an easy task. Hence the interest of recently described autoantibodies such as anti-pituitary autoantibodies that can be detected in celiac disease patients with low plasmatic levels of insulin-like growth factor 1(IGF-1). And, of course, antibodies against other tissue transglutaminase family members (i.e. anti-TG3 and anti-TG6) found in celiac disease or CD associated conditions such as gluten ataxia.
These are only some examples to illustrate the point of advances and future expectations in the sphere of celiac disease diagnose.

However, despite these and other exciting findings that will help, no doubts, to earlier and faster diagnoses of the disease in any circumstances, we are far away from the real understanding of the mechanisms underlying the disease and hence, from understanding factual therapies for it. Some old and apparently simple questions are yet awaiting to be solved out, such as the cofactor(s) enabling the disease to progress, or the cofactor braking it, in HLADQ2/8 individuals; the inability of the immune system to keep an homeostatic equilibrium in the gut of our patients remains deeply unknown; other points such as whether or not the disguised immune response triggered upon gluten ingestion in the susceptible individuals, is the cause or the consequence of the disease remains unanswered and requires a huge and internationally coordinated effort in basic research. Indeed, our progress in the ability to recognize the disease has stride but has not been accompanied by equivalent advances in the phisyopathogeneic knowledge nor in the therapeutical territories. Therefore, I will bet my money for basic research in celiac disease in the years to come, which would be definitely a priority, and the responsibility of the concerned scientific community in advances countries. This should be the way to change the famous definition of celiac disease as "tricky to find, hard to treat and impossible to cure" [1] by a new one, "easy to find, easy to treat and possible to cure" and then, if we manage to get it, I wish you were there.

\section{References}

[1] Lohiniemi, S., "Coeliac disease. Tricky to find, hard to treat, impossible to cure,” Lancet, 358(suppl.), S14, Dec 2001. 